\title{
Relación entre la potencia y velocidad en press de banca y la velocidad de lanzamiento de balón en jugadores profesionales de balonmano Relationship between barbell power and velocity in bench press exercise and ball throwing velocity in professional handball players \\ * Iker Javier Bautista, *Juan Vicente-M ampel, *Luis Baraja-Vegas, **Isidoro Martínez *U niversidad Católica deValencia (España), **U niversidad de León (España)
}

Resumen. Los objetivos de este estudio fueron (a) analizar la relación existente entre la una repetición máxima (1-RM) en press de bancay lavelocidad de lanzamiento en jugadores de balonmano U 18 denivel internacional y, (b) analizar qué variables del ejercicio del press de banca son más relevantes en el rendimiento específico (velocidad de lanzamiento del balón) durante el test de velocidad de lanzamiento $\left(\mathrm{T}_{3-\text {-steo }}\right.$ ). Dieciséis jugadores de la Selección Española de Balonmano Juvenil participaron en la presente investigación. Todos los sujetos realizaron un protocolo incremental en el ejercicio del press de banca, además del $T_{3-\text { stee }}$ de velocidad de lanzamiento del balón. Por un lado, se analizó la relación existente entre lavelocidad media(Vel ${ }_{\text {media }}$, velocidad media de la fase propulsiva $\left(\mathrm{Vel}_{\text {MFp }}\right)$, velocidad pico $\left(\mathrm{Vel}_{\text {pico }}\right.$ ), potencia media ( Pot $_{\text {media }}$ ), potencia media de la fase propulsiva $\left(\right.$ Pot $_{\text {MFP }}$ ), y potencia pico ( Pot $_{\text {pico }}$ ) en todo el espectro de cargas en relación con lavelocidad de lanzamiento. También se realizaron los mismos análisis con la carga en donde se obtuvo la máxima potencia media (Carga ${ }_{\mathrm{MP}}$ ). Los resultados

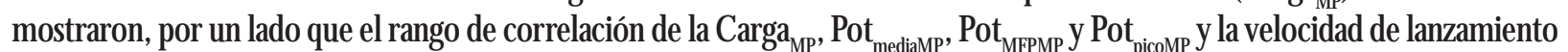
fueron de .61 ( $p=.012), .702(p<.01), .734(p<.01)$ y .63 $(p<.01)$, respectivamente. El coeficiente de correlación de Pearson entre la 1-RM y la velocidad de lanzamiento fue de $r=.61(p<.01)$. En conclusión, las variables relevantes a nivel

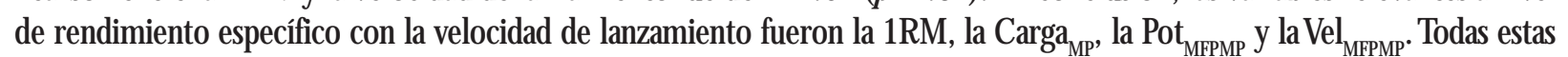
analizadas en función del $60 \%$ de la 1-RM.

Palabras clave: balonmano, potencia media fase propulsiva, velocidad media fase propulsiva, press de banca, velocidad de lanzamiento del balón.

\begin{abstract}
The objectives of this study were (a) to analyze the relationship between one repetition maximum (1-RM) in free bench press exercise and ball throwing velocity in handball players U 18 of international level and, (b) to analyze which variables of bench press exercise are more relevant in the specific performance during the ball throwing velocity test $\left(T_{3-\text { Step }}\right)$. Sixteen ( $n=16$ ) players of the SpanishYouth Handball Team participated in the present investigation. All subjects included performed an incremental protocol bench press exercise, in addition to the ${ }_{3-\text { Step }}$. $\mathrm{n}$ theone hand, it analyzed the relationship

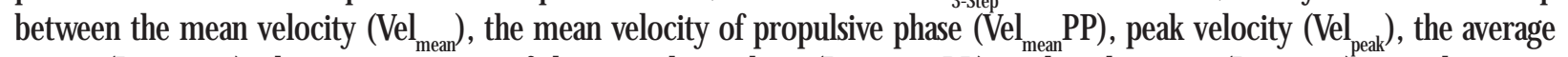
power (Power ${ }_{\text {mean }}$ ), the average power of the propulsive phase (Power $\left.{ }_{\text {mean }} \mathrm{PP}\right)$, and peak power (Power $\left.{ }_{\text {peak }}\right)^{\text {peak }}$ over the entire spectrum of charges in relation to the launch speed. The same analyzes were al so obtained with the load where the maximum average power $\left(\operatorname{Load}_{M P}\right)$. The results obtained, on the one hand that the correlation range of the $\operatorname{Load}_{M P}$, Power $_{\text {mean }} P$, Power $_{\text {MPP }} M$ P and Power ${ }_{\text {peek }} P$ P and ball throwing velocity were. $61(p=.012), .70(p<.01), .73(p<.01)$ and $0.63(p<.01)$, respectively. The correl ation coefficient between the $1-R M$ and ball throwing velocity was $r=0.61(p<.01)$. In conclusion, the relevant variables at the specific performance level with the ball throwing velocity were 1-RM, Load $_{M P}$, Power $_{M F P} M P$ and Vel ${ }_{M F P} M P$. All these analyzed according to $60 \%$ of the 1-RM .
\end{abstract}

Key words: handball, mean power of propulsive phase, mean velocity of propulsive phase, bench press exercise, ball throwing velocity.

\section{Introducción}

El balonmano es un deporte de alta intensidad y esfuerzos cortos y repetidos en el que habilidades como el salto, la carrera y el lanzamiento del balón pueden determinan el resultado final de un encuentro (Bautista

Fecha recepción: 13-08-20. Fecha de aceptación: 12-10-20 Iker Javier Bautista

ikerugr@gmail.com et al., 2016; Saavedra et al., 2018; Taylor, W right, Dischiavi, Townsend, \& M armon, 2017). Además de las habilidades técnico-tácticas, se ha argumentado que uno de los factores clave en el rendimiento es la velocidad delanzamiento del balón (Gorostiaga, Granados, Ibanez, \& Izquierdo, 2005) y el nivel de concentración (García, M oreno, Reina, \& M enayo, 2015). A diferencia de otros deportes, como el fútbol y el rugby, la cantidad de lanzamientos a portería que se producen durante un en- 
cuentro de balonmano es mayor. Por otro lado, en la mayoría de las ocasiones la distancia a portería a la que se produce el lanzamiento es inferior a nueve metros por lo que aquellos jugadores que lancen a mayor velocidad tendrán un potencial de rendimiento superior.

Generalmente, para evaluar la velocidad de lanza miento del balón en balonmano se utiliza el test de 3 pasos ( $T_{3-\text { Stee }}$ ) (Chelly, Hermassi, \& Shephard, 2010; Granados, Izquierdo, Ibanez, Bonnabau, \& Gorostiaga, 2007; Marques, Van Den Tillaar, Vescovi, \& GonzálezBadillo, 2007). A nivel de rendimiento deportivo, se han investigado diversos métodos y medios de entrenamiento para mejorar la velocidad de lanzamiento del balón, ya sea en balonmano o en otros deportes como el béisbol, el waterpolo o la disciplina de la jabalina (Van denTillaar, 2004). Desde un punto de vista científico, se han realizado investigaciones para comprobar el efecto sobre la velocidad de lanzamiento con balones sobre pesados-lastrados (DeRenne, Buxton, Hetzler, \& Ho, 1994), balonespor debajo desu peso normal (Van Muijen, Joris, Kemper, \&Van Ingen Schenau, 1991), con combinaciones deambosmétodos(DeRenne, Ho, \& Blitzblau, 1990) o con entrenamiento con resistencias externas (Souhail Hermassi, Chelly, Tabka, Shephard, \& Chamari, 2011; M cEvoy \& N ewton, 1998). Centrándonos en este último entrenamiento, los ejercicios por excelencia para mejorar la velocidad de lanzamiento son el press de banca y el pullover. En este sentido, la principal ventaja de estos tipos de ejercicios es la gran transferencia con el gesto del lanzamiento, ya que la musculatura implicada es similar a la musculatura que implica el gesto del lanzamiento (Fleck et al., 1992).

Recientemente, Hermassi, Schwesig, Aloui, Shephard, and Chelly (2019) han investigado sobre el efecto de un programa bisemanal de 8 semanas de duración sobre el lanzamiento del balón en jugadores de balonmano. Las sesiones de entrenamiento se realizaron con un volumen de tres o cuatro series y una intensidad entre el $75 \%$ y el $90 \%$ de la una repetición máxima (1-RM). Los principal es ejercicios utilizados fueron el press de banca, pullover y la arrancada. Los resulta dosobtenidosmostraron quelavelocidad de lanzamiento de balón se vio mejorada en el grupo experimental con un gran tamaño del efecto (TE = 2.78). Sin embargo, el grupo control no mejoró la velocidad de lanzamiento tras la aplicación del programa de entrenamiento. Por lo tanto, los autores de dicho trabajo concluyeron quela inclusión de ejercicios de levantamiento de pesas en jugadores de balonmano fue positiva debido a la explosividad y potencia que se requieren dichos ejerci- cios. Desafor tunadamente, en dicha investigación no se describieron las velocidades de ejecución en los principales ejercicios utilizados (i.e., press de banca y pullover). En cambio, un reciente estudio de Cuevas-Aburto, Janicijevic, Perez-Castilla, Chirosa-Ríos, \& GarcíarRa mos (2020) analizaron el efecto de dos programas de entrenamiento (i.e., cargas < 40\% 1-RM [balístico] vs. cargas entre el 70-90\% 1-RM [fuerza-potencia]) sobre la velocidad de ejecución en $20 \mathrm{~kg}$, la 1-RM en press de banca y la velocidad de lanzamiento del balón en juga dores no profesionales de balonmano. Tras 8 semanas de intervención los resultados mostraron que aquellos jugadores que realizaron el entrenamiento por debajo del $40 \%$ de la 1-RM mejoraron la velocidad de desplazamiento de la barra en press de banca en la intensidad de $20 \mathrm{~kg}[\mathrm{TE}=.63]$, pero no mejoraron la 1-RM [TE= .27] ni la velocidad de lanzamiento del balón. Por otro lado, aquellos jugadores que entrenaron entre el 70 y el $90 \%$ de la 1-RM, mejoraron el valor de la 1-RM [entre $2.6-7.2 \mathrm{~kg}]$, pero no mejoraron la velocidad de desplazamiento en $20 \mathrm{~kg}$ [TE = .21] ni la velocidad de lanzamiento del balón. Por otro lado, no se encontraron asociaciones significativas entre los cambios en la 1-RM vs. los cambios en la velocidad de lanzamiento del balón $(r=-.06, p=.748)$. Los autores del presente trabajo llegaron a la conclusión que un programa de corta dura ción basados únicamente en el ejercicio del press de banca, ya sea con cargas altas vs. cargas bajas, no resultó efectivo para la mejora de la velocidad de lanzamiento del balón. Las principales diferencias entre este estudio y el realizado por Hermassi et al. (2019), son tanto a nivel de intervención (i.e., además del press de banca, se incluyeron otrosejercicioscomo el pullover y laarrancada) como el nivel de los jugadores (i.e., profesionales vs. no profesionales).

La relación existente entre el ejercicio del press de banca y la velocidad de lanzamiento ha sido analizada en multitud de estudios (Chelly et al., 2010; Marques et al. , 2007). Generalmente, las variables utilizadas realizar los análisis son la velocidad o la potencia pico (Marques et al., 2007). Sin embargo, en otros estudios se han utilizado las variables de la velocidad y potencia media (Granados et al., 2007). La potencia y/ o velocidad media se calcula teniendo en cuenta todos los valores del rango de movimiento. En cambio, la potencia y/ o velocidad media de la fase propulsiva se calcula teniendo en cuenta los valores de potencia y/ o velocidad mediahastaquelafuerzaesmenor alg (Sanchez-M edina, Perez, \& Gonzalez-Badillo, 2010). En la literatura científica se ha argumentado que este val or es preferible a 
otros valores para medir el rendimiento específico en el ejercicio del press de banca (Sanchez-M edina $\&$ González-Badillo, 2011). Aunque diversos estudios muestran que la velocidad media y/ o pico es más esta ble en la medida en comparación a la velocidad media de la fase propulsiva en el ejercicio del press de banca (García-Ramos, Haff, Padial, \& Feriche (2018). En este sentido y atendiendo a la mecánica del lanzamiento, parece más correcto realizar los análisis correlaciónales entre las variables relevantes del ejercicio del press de banca y la velocidad de lanzamiento teniendo en cuenta los valores de la velocidad media de la fase propulsiva. Sin embargo, esta cuestión se encuentra aun sin resolver.

Una vez analizados todos los antecedentes, el objetivo de este estudio fue (a) analizar la relación existente entre el ejercicio del press de banca en peso libre y la velocidad de lanzamiento y (b) analizar que variables del ejercicio del press de banca son más relevantes en el rendimiento específico durante el test de velocidad de lanzamiento en jugadores de balonmano juveniles de nivel internacional. Todos estos análisis se han real izado desde dos perspectivas, teniendo en cuenta la carga a nivel absoluto 0 analizada en función de la máxima potencia en relación con la 1-RM.

\section{Material y método}

\section{Sujetos}

Un total de dieciséis jugadores juveniles de la Selección Española de Balonmano participaron en la presente investigación. La edad, peso, altura de los sujetosfueron de $18 \pm 0.4$ años, $87.4 \pm 9.97 \mathrm{~kg}$ y $188.9 \pm 6.72$ $\mathrm{cm}$, respectivamente. Todos los sujetos tenían una experiencia en el entrenamiento de balonmano de más de 6 años, además fueron considerados de élite porque: 1) La mayoría de los deportistas competían en la liga ASO BAL, 2) todos los deportistas formaban parte de la Selección Española de Balonmano y 3) la mayoría de ellos habían disputado la competición de Champion League con sus respectivos equipos. Todos los sujetos firmaron un consentimiento informado en donde se les explicó los riesgos y beneficios de la participación en la investigación. Este estudio fue revisado y aprobado por el comité ético de la Universidad.

\section{Procedimiento}

Todas las evaluaciones se llevaron a cabo durante una concentración del equipo para la preparación del campeonato del mundo Juvenil de Balonmano. Las eva luaciones se llevaron a cabo en el lugar habitual de entrenamiento durante el periodo de concentración. En primer lugar, se realizó el test para evaluar la velocidad de lanzamiento del balón. Mientras que el test incremental para la obtención de la 1-RM en el ejercicio del press de banca se realizó al siguiente día, tras 12 horas de recuperación.

Test de lanzamiento de balón en balonmano. La velocidad de lanzamiento del balón fue evaluada en una pista cubierta. Todos los sujetos realizaron un cal entamiento estandarizado de 10 minutos, en el cual se incluyó ejercicios de movilidad articular (i.e., tronco, hombros y muñecas) y desplazamientos por la pista a diferentes velocidades. Posteriormente, se midió la velocidad de lanzamiento de balón con una carrera de tres pasos $\left(\mathrm{T}_{3-\text { step }}\right)$. El sujeto debía mantener un pie en el suelo en el momento del lanzamiento. Cada deportista utilizó su mano dominante (definida como el brazo con el que lanza habitualmente) y su propia técnica personal. Paralarealización del test se utilizó un bal ón estándar de balonmano (i.e., $480 \mathrm{~g}$ de peso y $58 \mathrm{~cm}$ de circunferencia). Todos los sujetos realizaron un total de tres intentos, con un tiempo de al menos dos minutos de recuperación entre cada intento. Para la medición de la velocidad pico del balón se utilizó una pistola radar (Stalker ATS System, Radar Sales, Minneapolis, MMN, USA). El radar fue colocado detrás de la portería. Justo después de realizar cada lanzamiento se informó al deportista de la velocidad al canzada. El mejor valor de todos los intentos fue utilizado para los análisis estadísticos. El coeficiente de variación (CV) para la velocidad de lanzamiento del balón fue de $5.2 \%$.

Protocolo incremental de cargas en press de banca. Al día siguiente de la evaluación del $\mathrm{T}_{3 \text {-step' }}$ se realizó un protocolo incremental de cargas en el ejercicio del press de banca libre. Todos los sujetos estaban familiarizados con la técnica del ejercicio ya que realizaban habitualmente el press de banca en sus respectivos equipos. Los sujetos realizaron un calentamiento previo de 10 minutos además de realizar un total de dos series con una carga ligera (i.e., peso de la barra) antes de realizar el protocolo. El protocoló comenzó con una carga de $25 \mathrm{~kg}$, y se produjeron aumentos de $10 \mathrm{~kg}$, hasta llegar a una carga de $85 \mathrm{~kg}$. Posteriormente, el valor de la 1-RM fue calculado de manera individual incrementando la carga hastaqueel deportista sólo pudo realizar una repetición. Un tiempo de recuperación de cuatro minutos fue dejado entre cada serie. Se realiza ron un total de tres repeticiones en cada una de las intensidades hastallegar a los $85 \mathrm{~kg}$ En intensidades supe- 
riores a $85 \mathrm{~kg}$ se realizaron una y/ o dos repeticiones. Durante el protocolo incremental se le pidió al sujeto que real izaralafase excéntrica de forma controlada(i.e., a una velocidad baja y controlada). M ediante instrucciones del investigador se instó a cada sujeto a que debía levantar la barra lo más rápida posible (i.e. de forma explosiva), hasta llegar a una posición completamente extendida de los codos. De esta forma se evitó la realización de cualquier rebote de la barra contra el pecho. Para la evaluación de la velocidad media $\left(\mathrm{Vel}_{\text {media }}\right)$, velocidad media de la fase propulsiva $\left(V_{\text {MFP }}\right.$ ), velocidad pico $\left(V_{\text {pico }}\right)$, potencia media $\left(\right.$ Pot $\left._{\text {media }}\right)$, potencia media de la

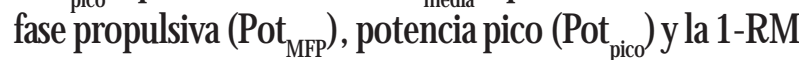
se utilizó un dispositivo de desplazamiento lineal (TForce System, Ergotech, Murcia, España). Este dispositivo consiste en un cable que se engancha a la barra. La información de la velocidad del movimiento es transmitida y transformada de una señal analógica a digital mediante una tableta de adquisición de datos. Posteriormente, los datos son analizados en un software customizado. Para el cálculo de cada una de las variables se tuvo en cuenta el promedio de las 3 repeticiones realizadas durante la realización del protocolo. Posteriormente, se seleccionó de forma individual la carga donde cada sujeto obtuvo la máxima potencia media del protocolo incremental $\left(\mathrm{Carga}_{\mathrm{MP}}\right)$. En dicha carga, se procedió al cál culo de las variables de la potencia media (Pot ${ }_{\text {media }}$ MP), potencia media de la fase propulsiva $\left(\right.$ Pot $\left._{\text {MFP }} M P\right)$, potencia pico (Pot $\left.{ }_{\text {pico }} M P\right)$, velocidad media ( Vel ${ }_{\text {media }}$ MP), velocidad media de la fase propulsiva $\left(V_{\text {MFP }} M P\right)$ y velocidad pico (Vel $\left.l_{\text {pico }} M P\right)$.

\section{Análisis estadísticos}

Todas las variables fueron expresadas como media \pm desviación estándar (SD). Todas las variables cumplieron el supuesto de normalidad (i.e., Shapiro-W ilks, $p>$.05). Para analizar la relación entre las variables relevantes del ejercicio del press de banca y el $\mathrm{T}_{3 \text {-step }}$ fue utilizada la correlación de Pearson. La magnitud del coeficiente de correlación de Pearson fue interpretada como: 0- . 10 (insignificante), .10- . 30 (pequeña), .30.50 (moderada), .50- .70 (grande), . .70- .90 (muy grande) y.9-1 (perfecta) (Hopkins, 2002). El coeficiente de variación (CV) fue cal culado como la desviación estándar dividido por la media multiplicado por 100. Las diferencias estadísticas se establecieron a nivel de $p<0.05$. Todos los análisis fueron realizados utilizando un paquete de análisis estadístico (SPSS, Inc , USA).

\section{Resultados}

En laTabla 1 se resumen los estadísticos descriptivos (i.e. media \pm SD) paralas variables dela 1-RM, Carga ${ }_{M P}$ $\mathrm{Pot}_{\text {media }} M P, \mathrm{Pot}_{\mathrm{MFP}} \mathrm{MP}, \mathrm{Pot}_{\text {pico }} \mathrm{MP}, \mathrm{Vel}_{\text {media }} M P, \mathrm{Vel}_{\mathrm{MFP}} \mathrm{MP}$ y Vel ${ }_{\text {pico }} M P$.

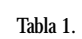

Análisis descriptivo (media y desviación estándar) de todas las variables de potencia y velocidad en la carga de la máxima potencia ( $C$ arga $a_{\mathrm{p}}$ ) en el ejercicio del press de banca.

$\frac{\text { en la carga de la maxima potencia (Cargamp) en el ejercicio del press de banca. }}{1-}$ \begin{tabular}{ccccccccc} 
& $(\mathrm{kg})$ & $(\mathrm{kg})$ & & & & $(\mathrm{m} / \mathrm{seg})$ & $(\mathrm{m} / \mathrm{seg})$ & $(\mathrm{m} / \mathrm{seg})$ \\
\hline Media & 97 & 58 & 461 & 541 & 935 & 0.84 & 0.85 & 1.42 \\
& 9 & 6.32 & 50 & 68 & 111 & 0.09 & 0.10 & 0.15
\end{tabular} \begin{tabular}{lllllllll} 
SD & 9.96 & 6.32 & 50 & 68 & 111 & 0.09 & 0.10 & 0.15 \\
\hline *1-RM $=$ valor de una repetición máxima; Carga & $=$ carga donde se obtuvo la máxima
\end{tabular} potencia media de todo el espectro de cargas analizado: Pot MP = valor de potencia media potencia mediade todo e exectro de cargas analizado; Pot media $\mathrm{MP}=$ va do de potencia meda de la fase propulsiva en la carga donde se obtuvo la maxima potencia, Pot pico $M P=$ valor de potencia pico en la carga donde se obtuvo la máxima potencia; $\mathrm{Ve}_{\text {media }} \mathrm{MP}=$ = valor de velocidad media en la carga donde se obtuvo la máxima potencia; Vel ${ }_{\text {media }} \mathrm{MP}=$ valor de velocidad media de la fase propulsiva en la carga donde se obtuvo la máxima potencia; Vel $\left.\right|_{\text {pico }} \mathrm{MP}=$ valor de potencia pico en la carga donde se obtuvo la máxima potencia.

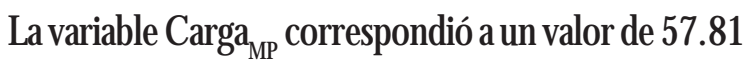
$\pm 6.32 \mathrm{~kg}$. Analizados en función de la 1-RM individual de cada participante, este valor correspondió al $60 \pm$ 4.74\%. La relación entre la $\mathrm{Carga}_{\mathrm{MP}}$ la Pot $_{\text {media }} \mathrm{MP}$, la Pot $_{\text {MFP }}$ MP y la Pot pico $_{\text {M }}$ y la velocidad de lanzamiento del balón fue de $r=.61(p=.012), r=.70(p<.01), r=$ $.73(p<.01)$ y $r=.63(p<.01)$, respectivamente (ver Figura 1). La relación entre la 1-RM y la velocidad de lanzamiento mostró un valor grande y estadísticamente significativo $(r=.61, p<.01)$.

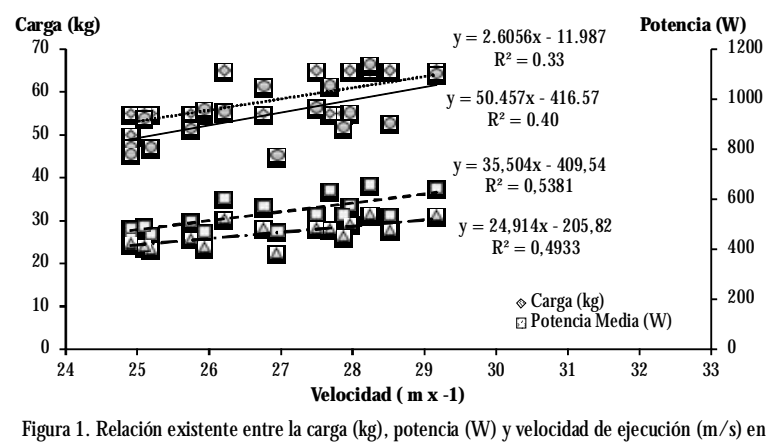
el ejercicio del press de banca.

En laTabla 2 se resumen los coeficientes de correla ción de Pearson entre las variables de potencia de todo el espectro de cargas analizadas en relación con la velocidad de lanzamiento del balón.

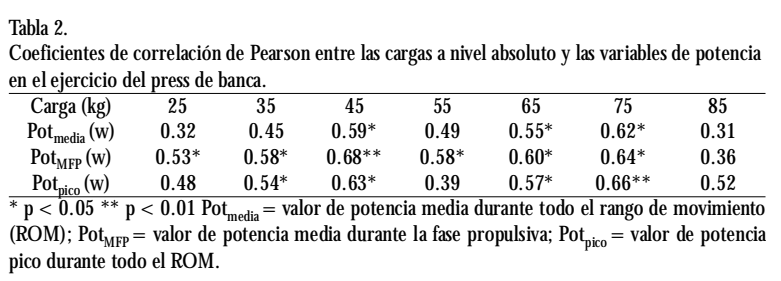

En laTabla 3 se resumen los coeficientes de correla ción de Pearson entre las variables de velocidad de todo el espectro de cargas analizadas en relación con la velocidad de lanzamiento del balón. 
Tabla3.

Coeficientes de correlación de Pear son entre las cargas a nivel absoluto y las variables de

\begin{tabular}{ccccccc} 
velocidad en el ejercicio del press de banca. \\
\hline Carga (kg) & 25 & 35 & 45 & 55 & 65 & 75
\end{tabular}

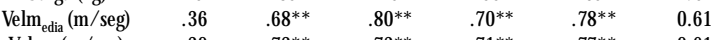

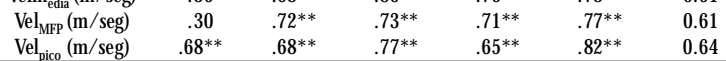
$* p<0.05^{* *} p<0.01$ Pot $_{\text {media }}=$ valor de potencia media durante todo el rango de movimiento; Pot $_{\text {MFP }}=$ valor de potencia media durante la fase propulsiva; Pot $_{\text {pico }}=$ valor de potencia pico durante todo el rango de movimiento.

Los resultados de la correlación de Pearson entre la $\mathrm{Vel}_{\text {media }} \mathrm{MP}, \mathrm{Vel}_{\text {MFP }} \mathrm{MP \text {PlaVel}}$ pico $\mathrm{MP}$ y la velocidad de lanzamiento fueron de $r=.35(p=.177), r=.59(p<.05)$ $y r=.24(p=.356)$, respectivamente.

\section{Discusión}

Para nuestro conocimiento, este es el primer traba jo que analiza la relación entre las variables como la Pot $_{\text {MFp }}$ y Vel $\mathrm{MFp}_{\text {MF }}$ en el ejercicio del press de banca y la velocidad de lanzamiento en jugadores profesionales de balonmano. Por un lado, se han analizado la dinámica de las variables descritas en relación con la carga en donde se obtuvo la Carga $a_{\text {MP }}$ (i.e., 60\% 1-RM) de todo el espectro de intensidades. Mientrasque por otro lado, este análisis se ha realizado teniendo en cuenta las cargas a nivel absoluto (i.e., desde 25 hasta $85 \mathrm{~kg}$ ). Los resulta dos obtenidos mostraron que los valores de la 1-RM y la Carga se relacionaron moderadamente y de forma estadísticamente significativa con la velocidad de lanza miento del balón.

Como ya se había mostrado en estudios anteriores (Gorostiaga et al., 2005; Granados et al., 2007; Marques et al., 2007) existe una relación entre las variables de rendimiento del tren superior medidas en la fase concéntrica (i.e. potencia y velocidad en ejercicio del press de banca) con la velocidad de lanzamiento del ba lón. En este sentido, Marques et al. (2007) analizaron la relación en un protocolo de 3 cargas (26, 36 y $46 \mathrm{~kg}$ ) con la velocidad de lanzamiento del balón en jugadores de balonmano. Las variables evaluadas fueron la potencia y velocidad pico. En dicho estudio se encontró una relación significativa entre la potencia pico y la velocidad de lanzamiento de $r=0.59, r=0.63$, para las cargas de 36 y $46 \mathrm{~kg}$, respectivamente. En nuestro estudio, los resultados del coeficiente de correlación entre la potenciay velocidad pico con lavelocidad de lanzamiento fueron de $r=.54, r=.63 \mathrm{y} r=.68, r=.77$, para las cargas absolutas de 35 y $45 \mathrm{~kg}$, respectivamente (ver Tabla 2 y 3$)$.

Por otro lado, observando la1-RM en nuestramuestra este valor fue de $97 \pm 9.96 \mathrm{~kg}$ Este resultado fue superior al encontrado por Ingebrigtsen, Jeffreys, and Rodahl (2013) en 29 jugadores U 18 talentosos que fue de $75 \pm$
$13.7 \mathrm{~kg}$. En el estudio realizado por Granados et al. (2007) encontraron un coeficiente de correlación entre la 1-RM y la velocidad de lanzamiento del balón de $r=$ 0.61 . La muestra utilizada fueron 16 chicas pertenecientes a un club de máximo nivel en balonmano. Los resultados obtenidos en nuestra investigación concuerdan con losvalores presentadosen el estudio de Granados et al. (2007). En concreto, el coeficiente de correlación entre la 1-RM y la velocidad de lanzamiento fue de $r=$ $.61(p<.05)$. Del mismo modo, en el estudio de Marques et al. (2007) el coeficiente de correlación entre dichas variables fue de $r=.64$. En esta ocasión, la muestra utilizada fueron varones pertenecientes a un club de máximo nivel. Por su parte, Chelly et al. (2010), utilizando una muestra total de 14 jugadores de balonmano, encontraron un correlación positiva entre la 1-RM y la velocidad de lanzamiento de $r=.56$. Por lo tanto, según resultados obtenidos cabría destacar la importancia de la fuerza dinámica máxima para obtener un alto nivel de rendimiento en el gesto específico del lanzamiento en apoyo clásico, ya que ambas variables tienen alrededor de un 36\% de la varianza compartida. En este sentido, en el estudio de Granados et al. (2007) observaron como la variable de la 1-RM mejoró un $1.6 \%$ a lo largo de una temporada de balonmano en jugadores varones profesionales. Según se define en Van den Tillaar (2004) el gesto específico del lanzamiento puede mejorarse a través de entrenamientos basados en la mejora de la fuerza, de la velocidad y/ 0 entrenamientos basados en la especificidad del gesto.

Por otra parte, aunque el valor de la 1-RM se ha mostrado que tiene una asociación positiva y significativa con la velocidad de lanzamiento del balón en jugadores de bal onmano, cuando se anal izan las cargas a nivel absoluto en un amplio espectro de intensidades podemos comprobar la importancia que tienen las variables como la potencia y velocidad de ejecución en todo el espectro de cargas. Estudios previos han mostrado la asociación existente entre la potencia y velocidad pico (M arques et al., 2007). Espećficamente, en nuestra investigación hemos realizado los análisis desde dos puntos de vista (i.e., a nivel absoluto y teniendo en cuenta la $\mathrm{Carga}_{\mathrm{MP}}$ ) y con 3 variables diferentes (i.e., Pot ${ }_{\text {media' }}$ la

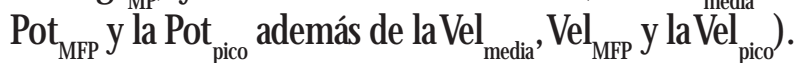
A nivel absoluto podemos destacar que los coeficientes de correlación entre dichas variables (i.e., potencia y velocidad) y la velocidad de lanzamiento en todo el rango de cargas analizado fueron entre pequeños y muy grandes, en función de la carga analizada (ver Tabla 2 y 3). Sin embargo, cabe destacar que las variables de la 
Pot $_{\text {MFP }}$ y la Vel $\mathrm{MFP}_{\text {mF }}$ obtuvieron un mejor coeficiente de correlación, tanto a nivel absoluto como a nivel de la Carga $_{\mathrm{MP}}$, con la velocidad de lanzamiento del balón ( $r=$ .73 y $r=0.59$, para las variables Pot ${ }_{M F P} M P$ ) y Vel ${ }_{M F P} M P$, respectivamente). U na posible explicación a este fenómeno podría ser a la relación específica entre la fase propulsiva del gesto del press de banca con la mecánica del lanzamiento en balonmano. Para realizar los cálculos de la fase propulsiva se tienen en cuenta los val ores hasta que la fuerza ejercida en la barra es igual a $1 \mathrm{~g}$, es decir, sin tener en cuenta la fase de frenado. Durante el lanzamiento en balonmano, las velocidades de los segmentos no son máximas, puesto que se busca un mínimo de precisión (i.e. intentar meter un gol en la portería).

Desde otro punto de vista, se ha encontrado una relación positiva y significativa entre la variable de la Carga $_{\mathrm{MP}}$ con la velocidad de lanzamiento $(r=.61, p<$ .05). De este modo, aquellos jugadores que en la varia ble de la Carga $_{\mathrm{MP}}$ levantaron más carga, mayor fue su velocidad de lanzamiento del balón en el test específico realizado. Este factor resulta importante para el entrenamiento puesto que uno de los objetivos que nos planteamos cuando realizamos entrenamiento con resistencias externas, no es sólo mejorar el máximo valor de la 1-RM, si no mejorar la velocidad y potencia de desplazamiento en cada una de las partes de la curva de fuerzavelocidad. Hermassi et al. (2011) encontraron un aumento significativo en la sección transversal del músculo de ambos miembros en el tren superior tras 8 semanas de entrenamiento enfocado hacia la fuerza y la potencia. Dicho entrenamiento se llevó a cabo durante dos días por semana. De forma específica, tanto la 1RM como la potencia pico mejoraron de forma significativa en el grupo que realizó entrenamientos de fuerzaen el tren superior. Los resultadosobtenidos en nuestro estudio sugieren que además de conocer el valor de la 1-RM en el ejercicio del press de banca, resulta interesante conocer a que velocidad y potencia se desarrollan las intensidades submáximas. En este sentido, las varia bles de la Pot ${ }_{M F P}$ MP y laVel ${ }_{M F P}$ M P correlacionaron mejor con la velocidad de lanzamiento que las variables de la vel ocidad y potencia media o pico, tanto a nivel absoluto como a nivel relativo.

Una de las posibles limitaciones de nuestro estudio fue la escasa muestra utilizada. Sin embargo, cabe destacar que el nivel de los deportistas analizados era de nivel internacional. Dada las características del estudio (i.e., descriptivo-correlacional) no es posible establecer relaciones de causalidad. Es por ello, que futuras investigaciones necesitan ser realizadas para analizar si el incremento en algunas de estas variables se ven reflejados en cambios en el rendimiento en los test específicos a lo largo de un periodo de intervención. Y aun más importante, si la asociación entre los cambios de dichas variables tras un programa de intervención obtienen relaciones estadísticamente significativas.

Por un lado, la principal aplicación de este trabajo al campo práctico es que cuando se está evaluando el ejercicio del press de banca, además de laVel ${ }_{\text {pico }}$ o laVel ${ }_{\text {media' }}$ debemos de tener en cuenta otras variables como la

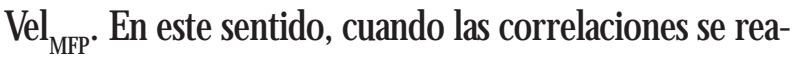

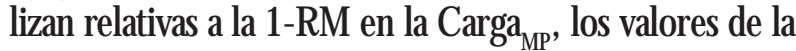

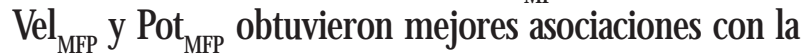
velocidad de lanzamiento. Por otro lado, aunque el valor de la 1-RM es un buen indicador de rendimiento en el test de velocidad de lanzamiento del balón, si se rea lizaun protocolo incremental de cargas en press de banca y además disponemos de un dispositivo de desplazamiento lineal, podemos obtener información muy relevante para la planificación y evaluación del entrenamiento deportivo.

\section{Conclusiones}

Por lo tanto, para concluir el presente estudio podemos señalar que existe una relación positiva y significa tiva entre el ejercicio del press de banca y la velocidad de lanzamiento en el $T_{3-\text { step }}$ Las variables relevantes a nivel de rendimiento específico en el ejercicio del press de banca con la velocidad de lanzamiento fueron la 1RM, la Carga $_{M P}$, la Pot MFP $_{\text {M P y laVel }}$ MPP M. Todas estas analizadas en función del $60 \%$ de la 1-RM. A nivel de cargas absolutas, se obtuvieron buenos coeficientes de correlación en todos los niveles de variables de potencia y velocidad.

\section{Agradecimientos}

Nos gustaría agradecer al cuerpo técnico de la Selección Española Juvenil de Balonmano (M édico, Fisioterapeuta y Delegado) por su ayuda a la hora de la recolección de los datos.

\section{Referencias}

Bautista, I. J., Chirosa, I.J., Robinson, J. E., van derTillaar, R., Chirosa, L. J., \& Martin, I. M. (2016). A new physical performance classification system for elite handball players: cluster analysis. J Hum Kinet, 51, 131-142. 
doi:10.1515/ hukin-2015-0177

Chelly, M. S., Hermassi, S., \& Shephard, R. J. (2010). Relationships between power and strength of the upper and lower limb muscles and throwing velocity in male handball players. The Journal of Strength \& Conditioning Research, 24(6), 1480-1487.

Cuevas-A burto, J., Janicijevic, D., Perez-Castilla, A., ChirosaRíos, L. J. \& \& García-Ramos, A. (2020). Changesin bench pressperformance and throwing velocity after strengthoriented and ballistic resistance training programs. The Journal of sports medicine and physical fitness, 10.23736/ S0022-4707.20.11011-9. Advance online publication. https:/ / doi.org/ 10.23736/ S0022-4707.20.11011-9

GarcíaH errero, J.A., M oreno Hernández, F. J., ReinaVállo, R., \& M enayoAntúnez, R. (2015). Lavelocidad y lapre cisión en el lanzamiento en jóvenes jugadores de balonmano en función de la concentración de la práctica (The speed and accuracy in the shot in young handball players according to the concentration of practice). Retos, (19), 43-46. https:// doi.org/ 10.47197/ retos. v0i19.34636

DeRenne, C., Buxton, B. P., Hetzler, R. K., \& Ho, K.W. (1994). Effects of under-and overweighted implement training on pitching velocity. The Journal of Strength \& Conditioning Research, 8(4), 247-250.

DeRenne, C., Ho, K., \& Blitzblau, A. (1990). Effects of weighted implement training on throwing velocity. The Journal of Strength \& Conditioning Research, 4(1), 16-19.

Fleck, S. J., Smith, S. L., Crab, M.W., Denahan, T., Snow, R. E., \& Mitchell, M. L. (1992). Upper extremity isokinetic torqueand throwing velocity in team handball. The Journal of Strength \& Conditioning Research, 6(2), 120-124.

GarcíarRamos, A., Haff, G. G., Padial, P., \& Feriche, B. (2018). Reliabil ity of power and velocity variables collected during the traditional and ballistic bench press exercise. Sports biomechanics, 17(1), 117-130. https:// doi.org/ 10.1080/ 14763141.2016.1260767

Gorostiaga, E., Granados, C. , Ibanez,J ., \& Izquierdo, M. (2005). Differencesin physical fitnessand throwing velocity among eliteand amateur malehandball players. International Journal of SportsM edicine, 26(03), 225-232.

Granados, C., Izquierdo, M., Ibanez, J., Bonnabau, H., \& Gorostiaga, E. (2007). Differences in physical fitnessand throwing velocity among elite and amateur female handball players. International Journal of Sports M edicine, 28(10), 860-867.

Hermassi, S., Chelly, M. S., Tabka, Z., Shephard, R. J., \& Chamari, K. (2011). Effects of 8-week in-season upper and lower limb heavy resistance training on the peak power, throwing velocity, and sprint performance of elite malehandball players. The ournal of Strength \& Conditioning Research, 25(9), 2424-2433.
Hermassi, S., Schwesig, R., Aloui, G., Shephard, R. J. , \& Chelly, M. S. (2019). Effects of Short-Term In-SeasonWeightlifting Training on the MuscleStrength, Peak Power, Sprint Performance, and Ball-ThrowingVelocity of M aleHandball Players. I Strength Cond Res, 33(12), 3309-3321. doi: $10.1519 /$ jsc. 0000000000003068

Hopkins,W. (2002). A scale of magnitudesfor effect statistics [20 03 2010]. Internet link: http:/ / sportsci. org/ resource/ statsl effectmag. html.

Ingebrigtsen, J., Jeffreys, I., \& Rodahl, S. (2013). Physical characteristicsand abilities of junior elitemal eand female handball players. The Journal of Strength $\&$ Conditioning Research, 27(2), 302-309.

Marques, M. C., Van DenT illaar, R.,Vescovi, J. D., \& GonzálezBadillo, J. J. (2007). Relationship between throwing velocity, muscle power, and bar velocity during bench pressin elite handball players. International journal of sports physiology and performance, 2(4), 414-422.

M cEvoy, K. P., \& N ewton, R. U. (1998). Baseball throwing speed and base running speed: The effects of ballistic resistance training The Journal of Strength \& Conditioning Research, 12(4), 216-221.

Saavedra, J. M., Kristjánsdóttir, H., Einarsson, I., Guðmundsdóttir, M. L., Porgeirsson, S., \& Stefansson,A. (2018). Anthropometric Characteristics, Physical Fitness, andThrowingVelocity in Elitewomen's H andballTeams. J Strength Cond Res, 32(8), 2294-2301. doi:10.1519/ jsc. 0000000000002412

Sanchez-M edina, L., \& González-Badillo,J.J. (2011).Velocity loss as an indicator of neuromuscular fatigue during resistance training M edicine $\&$ Science in Sports $\&$ Exercise, 43(9), 1725-1734.

Sanchez-M edina, L., Perez, C., \& Gonzalez-Badillo, J. (2010). Importance of the propulsive phasein strength assessment. International J ournal of Sports M edicine, 31(02), 123-129.

Taylor, J. B.,W right,A.A., Dischiavi, S. L., Townsend, M.A., \& Marmon, A. R. (2017). Activity Demands During MultiDirectionalTeam Sports: A Systematic Review. SportsM ed, 47 (12), 2533-2551. doi:10.1007/ s40279-017-0772-5

Van denTillaar, R. (2004). Effect of different training programs on the velocity of overarm throwing: abrief review. The Journal of Strength \& Conditioning Research, 18 (2), 388-396.

Van Muijen, A. E., Joris, H., Kemper, H. C., \& Van Ingen Schenau, G. J. (1991). Throwing practicewith different ball weights: Effects on throwing velocity and muscle strength in female handball players. Research in Sports Me dicine:An International Journal, 2(2), 103-113. 